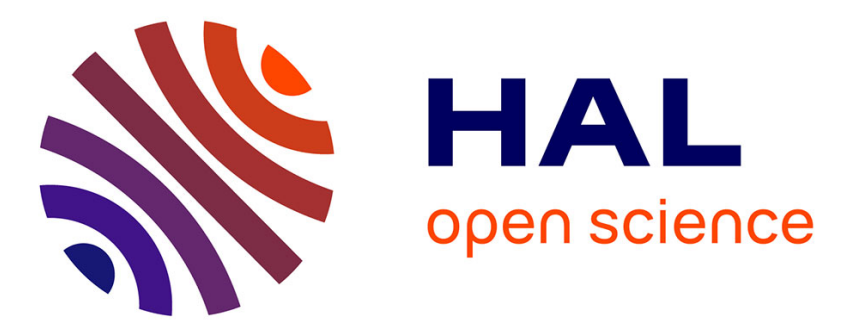

\title{
Modeling of Magnetic Induced Deformation Using Computer Code Chaining and Source Tensor Projection
}

Mingyong Liu, Zuqi Tang, Xavier Mininger, Frédéric Bouillault, Olivier Hubert, Laurent Bernard

\section{- To cite this version:}

Mingyong Liu, Zuqi Tang, Xavier Mininger, Frédéric Bouillault, Olivier Hubert, et al.. Modeling of Magnetic Induced Deformation Using Computer Code Chaining and Source Tensor Projection. IEEE Transactions on Magnetics, 2017, 53 (6), pp.1-4. 10.1109/TMAG.2017.2666421 . hal-01531198

\section{HAL Id: hal-01531198 \\ https://hal-centralesupelec.archives-ouvertes.fr/hal-01531198}

Submitted on 11 Mar 2020

HAL is a multi-disciplinary open access archive for the deposit and dissemination of scientific research documents, whether they are published or not. The documents may come from teaching and research institutions in France or abroad, or from public or private research centers.
L'archive ouverte pluridisciplinaire $\mathbf{H A L}$, est destinée au dépôt et à la diffusion de documents scientifiques de niveau recherche, publiés ou non, émanant des établissements d'enseignement et de recherche français ou étrangers, des laboratoires publics ou privés. 


\title{
Modeling of Magnetic Induced Deformation Using Computer Code Chaining and Source Tensor Projection
}

\author{
Mingyong Liu* ${ }^{*}$, Zuqi Tang*, Xavier Mininger*, Frédéric Bouillault* Olivier Hubert ${ }^{\dagger}$ and Laurent Bernard ${ }^{\ddagger}$ \\ ${ }^{*}$ GeePs, CNRS UMR 8507, CentraleSupelec, Univ. Paris-Sud, UPMC, 91192, France \\ ${ }^{\dagger}$ LMT-Cachan, CNRS UMR 8535, ENS-Cachan, Univ. Paris-Saclay, 94235, France \\ $\ddagger_{\text {GRUCAD/EEL/CTC, Universidade Federal de Santa Catarina, Florianpolis 88040-900, Brazil }}$
}

\begin{abstract}
Source tensor projections are developed for the magneto-elastic coupled problems when magnetostriction induced force and magnetic force are considered. Comparisons with classical force density projection are first performed on a simple example. Then it is investigated on an application of a multi-layer transformer core with the consideration of material anisotropy and multi-layer inhomogeneity.
\end{abstract}

Index Terms-Finite element method, Source tensor projection, Magnetostriction, Magnetic force, Magneto-elastic problem.

\section{INTRODUCTION}

Magneto-elastic coupled problem is usually solved by dividing it into two sub-problems: magnetic part and mechanic part [1], [2]. The magnetic resolution gives the distribution of magnetic field, leading to the computation of magnetic force and magnetostriction induced force. These forces are used as loadings of mechanical resolution, leading to structure deformations and vibrations. Each sub-problem can be solved on its own mesh with adapted support and optimized discretization. Such a modeling process requires the data projection from one mesh to another, making mesh-to-mesh date transfer crucial in these high accuracy multi-physics modelings.

Mesh-to-mesh projection can be performed through an explicit interpolation or Galerkin method [3], [4]. The latter has the advantage of great overall precision, and has already been applied in various domains such as fluid mechanics [5], thermo-mechanic [6] and magnetothermic [7]. For magneto-elastic coupled problems, Galerkin projections can be carried out at different stages. Parent et al. [7] show the possibility of projecting magnetic field, magnetic vector potential and force density. Wang et al. compare the magnetic field projection in different spaces [8], and propose energetic Galerkin projection [9] to minimize the errors of magnetic energy while projecting magnetic field. Journeaux et al. [10] demonstrate that, for a magneto-elastic coupled modeling chain, projection of magnetic force density offers better accuracy than projection of magnetic field.

However, for most of mechanical computation softwares, only source tensor (strain/stress) and force density are considered as source term. Projections of magnetic field or magnetic potential will be hard to achieve. Moreover, data transfer of magnetostriction induced force and deformation have never been studied in the literature. To tackle these issues, source tensor projection is developed for both magnetic force and magnetostriction induced force. They are then compared with classical force density projection on an isotropic ferromagnetic piece. Application on a laminated transformer core with material anisotropy and nonlinearity is finally achieved.

\section{Projection Theoretical Considerations}

Let us consider $\mathcal{D} \subset \mathbb{R}^{2}$ the studied domain. $L^{2}(\mathcal{D})$ is a space of square integrable vectorial or tensorial functions, depending on the context. Two function spaces are defined: $V_{1} \in L^{2}(\mathcal{D})$ in the source mesh; $V_{2} \in L^{2}(\mathcal{D})$ in the target mesh. The aim of the mesh-to-mesh projection is to find the fields in target mesh $u^{t} \in V_{2}$, which possess the minimum errors compared to the fields in source mesh $u^{s} \in V_{1}$.

By defining a test function in the target space $u^{\prime} \in V_{2}$, Galerkin method gives the formulation to solve $u^{t}$ in weak form. Equation (1) and (2) are adapted respectively to vectorial field and tensorial field ( $2^{\text {nd }}$-order).

$$
\begin{aligned}
\int_{\mathcal{D}} u^{s} \cdot u^{\prime} \mathrm{d} \tau & =\int_{\mathcal{D}} u^{t} \cdot u^{\prime} \mathrm{d} \tau \\
\int_{\mathcal{D}} u^{s}: u^{\prime} \mathrm{d} \tau & =\int_{\mathcal{D}} u^{t}: u^{\prime} \mathrm{d} \tau
\end{aligned}
$$

In discrete domain, $u^{s}$ and $u^{t}$ are usually defined as (3)

$$
u^{s}=\sum_{i=1}^{N_{s}} u_{i}^{s} \omega_{i}^{s} \quad \text { and } \quad u^{t}=\sum_{i=1}^{N_{t}} u_{i}^{t} \omega_{i}^{t}
$$

Source tensors and nodal forces are associated respectively to each element and node. $N_{s}$ and $N_{t}$ are the number of elements or nodes in $2 \mathrm{D}$ case. $u_{i}^{s}$ and $u_{i}^{t}$ are the degrees of freedom associate with the $i^{t h}$ node or element. $\omega_{i}^{s}$ and $\omega_{i}^{t}$ are the shape functions on $i^{\text {th }}$ node or element. By applying the classical Ritz-Galerkin method, the test function $u^{\prime}$ is naturally chosen to be the same as the shape function in the target mesh $u^{\prime}=$ $\left\{\omega_{i}^{t}\right\}_{i=1}^{N_{t}}$. This leads to a linear system as follows:

$$
[A]\left[U^{s}\right]=[B]\left[U^{t}\right]
$$

where $\left[U^{s}\right]$ and $\left[U^{t}\right]$ are vectors of degrees of freedom in source and target meshes. $[A]_{N_{t} \times N_{s}}$ and $[B]_{N_{t} \times N_{t}}$ are two matrices with their elements defined in (5).

$$
A_{i, j}=\int_{\mathcal{D}} \omega_{i}^{t} \omega_{j}^{s} \mathrm{~d} \tau \quad \text { and } \quad B_{i, j}=\int_{\mathcal{D}} \omega_{i}^{t} \omega_{j}^{t} \mathrm{~d} \tau
$$

\section{MOdeling CHAIN OF MAGNETO-ELASTIC PROBLEM}

\section{A. Formulation of magnetic induced deformation}

This weakly coupled magneto-elastic problem is solved using a sequential approach: magnetic resolution is followed by mechanical resolution. Studied domains of magnetic resolution is noted as $\mathcal{D}_{\text {mag }}$, including air and iron areas. $\mathcal{D}_{\text {mec }}$ is defined as a subdomain of $\mathcal{D}_{\text {mag }}\left(\mathcal{D}_{\text {mec }} \subset \mathcal{D}_{\text {mag }}\right)$, containing only the iron part, dedicated to the mechanical resolution. 
A simplified multi-scale model (SMSM) [11], [12] is involved, based on energetic description of the fictitious equivalent single crystal randomly oriented. Local magnetostriction strain $\epsilon_{\mu}^{\alpha}$ and magnetization $\vec{M}^{\alpha}$ are considered a function of orientation. The local potential energy of domain $\alpha$ consists of magnetic, elastic, anisotropy and surface energy. This leads to the volume fractions $f^{\alpha}$ of domain $\alpha$, using a Boltzmann type relation. Macroscopic behavior is obtained as an average of local behavior:

$$
\vec{M}=\sum_{\alpha} f_{\alpha} \vec{M}^{\alpha} \quad \text { and } \quad \epsilon_{\mu}=\sum_{\alpha} f_{\alpha} \epsilon_{\mu}^{\alpha}
$$

Static magnetic resolution is carried out based on scalar potential formulation. Distributions of magnetic field $\vec{H}$, induction $\vec{B}$ and free magnetostriction strain $\boldsymbol{\epsilon}_{\mu}$ are obtained. This leads to the computation of magnetic induced source tensors, and then relating forces.

Magnetic induced deformations are generated by two sources: magnetostriction induced forces and magnetic forces. These forces are computed from their source tensors in weak form, leading to nodal forces. Nodal force is a fictive force, numerically concentrated on the nodes. The magnetostriction induced nodal force $\vec{F}_{\mu}$ is obtained from the free magnetostriction strain $\epsilon_{\mu}$ as source tensor [12], with $\mathbb{C}$ the stiffness tensor of the medium.

$$
F_{\mu}^{u, i}=\int_{\mathcal{D}_{m e c}}\left(\operatorname{grad}_{s}\left(\vec{u}^{i}\right): \mathbb{C}: \boldsymbol{\epsilon}_{\mu}\right) \mathrm{d} \tau
$$

With $\vec{u}^{i}$ the vectorial test function of the node $i$, and $F_{\mu}^{u, i}$ the nodal equivalent force of the node $i$ in the direction of $\vec{v}$. The magnetic nodal force $\vec{F}_{m}$ is obtained from Maxwell stress tensor $\boldsymbol{T}$ as source tensor using the same principal.

$$
F_{m}^{u, i}=\int_{\mathcal{D}_{\text {mag }}}\left(\boldsymbol{g r a d}_{s}\left(\vec{u}^{i}\right): \boldsymbol{T}\right) \mathrm{d} \tau
$$

This Maxwell stress tensor is adapted to the possible nonlinear and anisotropic material behaviors [13], defined as follows:

$$
\boldsymbol{T}=\varphi(\vec{H}) \boldsymbol{\delta}-\frac{1}{2}(\vec{H} \otimes \vec{B}+\vec{B} \otimes \vec{H})
$$

where $\varphi(\vec{H})$ is the magnetic coenergy. $\delta$ is the unit matrix.

Once the nodal forces are computed, a classic linear elastic problem is ready to be solved [12].

$$
\left([K]+2 j \beta \omega^{n}[K]-\left(\omega^{n}\right)^{2}[M]\right)[U]=[F]
$$

where $[M]$ and $[K]$ are respectively the mass matrix and stiffness matrix. $\omega$ and $\beta$ stand for angular speed $(\mathrm{rad} / \mathrm{s})$ and damping coefficient. As we intend to study the best projection method for forces with different natures, $[F]$ stands for either array of magnetic nodal force or magnetostriction induced nodal force. $[U]$ is the array of degrees of freedom of the displacement field. From (10) the static elastic problem may be considered by setting $\omega=0$.

\section{B. Source Tensor Projection}

Both magnetic force and magnetostriction induced force are deduced form their source tensors: free magnetostriction strain and Maxwell tensor. It is believed that these source tensors

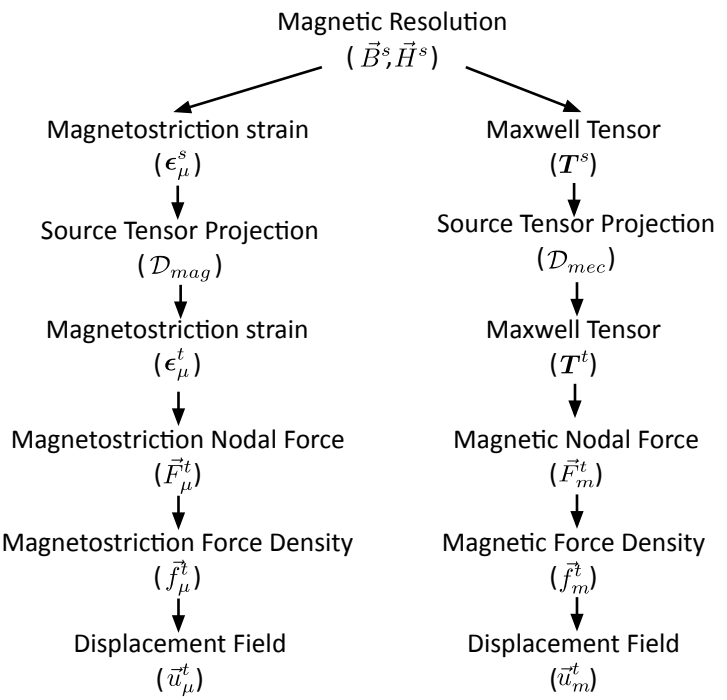

Fig. 1. Strategy of source tensor projection

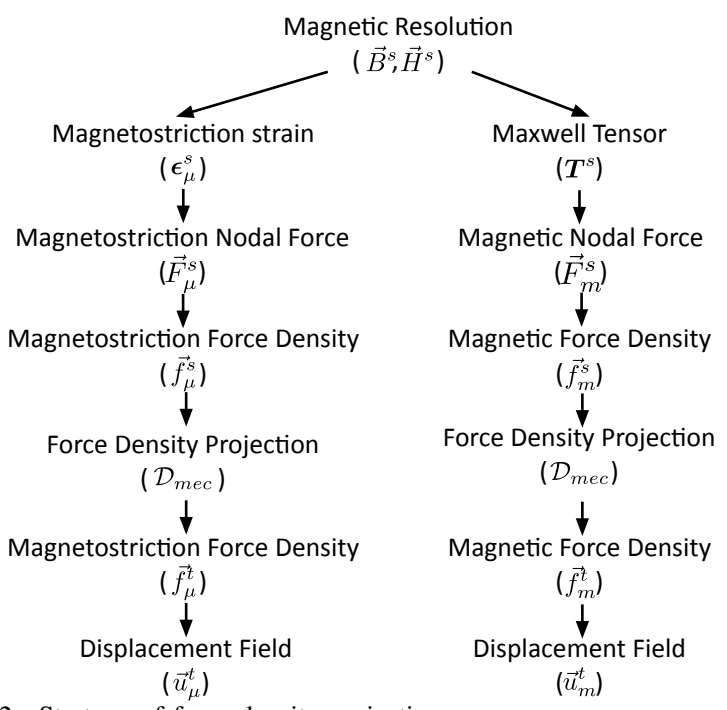

Fig. 2. Strategy of force density projection

contain more information than their associated nodal force vector. For sequential modeling chain, data projections can be carried out at source tensor stage. Given $\boldsymbol{\epsilon}_{\mu}^{s}, \boldsymbol{T}^{s}, \in V_{1}$, find $\boldsymbol{\epsilon}_{\mu}^{t}, \boldsymbol{T}^{t} \in V_{2}$, such that:

$$
\begin{aligned}
& \int_{\mathcal{D}_{m e c}} \boldsymbol{\epsilon}_{\mu}^{s}: \boldsymbol{\epsilon}_{\mu}{ }^{\prime} \mathrm{d} \tau=\int_{\mathcal{D}_{\text {mec }}} \boldsymbol{\epsilon}_{\mu}^{t}: \boldsymbol{\epsilon}_{\mu}{ }^{\prime} \mathrm{d} \tau, \quad \forall \boldsymbol{\epsilon}_{\mu}{ }^{\prime} \in V_{2} . \\
& \int_{\mathcal{D}_{\text {mag }}} \boldsymbol{T}^{s}: \boldsymbol{T}^{\prime} \mathrm{d} \tau=\int_{\mathcal{D}_{\text {mag }}} \boldsymbol{T}^{t}: \boldsymbol{T}^{\prime} \mathrm{d} \tau, \quad \forall \boldsymbol{T}^{\prime} \in V_{2} .
\end{aligned}
$$

Projections of free magnetostriction strain tensor and Maxwell tensor are carried out using the same formulation mentioned in (2). As Maxwell tensor is non-null in the air, the integration domain for (12) is carried out in $\mathcal{D}_{\text {mag }}$. This is different from the free magnetostriction strain, which exists only in the iron part. Force densities are then computed in the target mesh. The main procedure is summarised in Fig.1.

\section{Force density Projection}

Source tensor is not always available, because magnetic forces are often computed through virtual power principle 


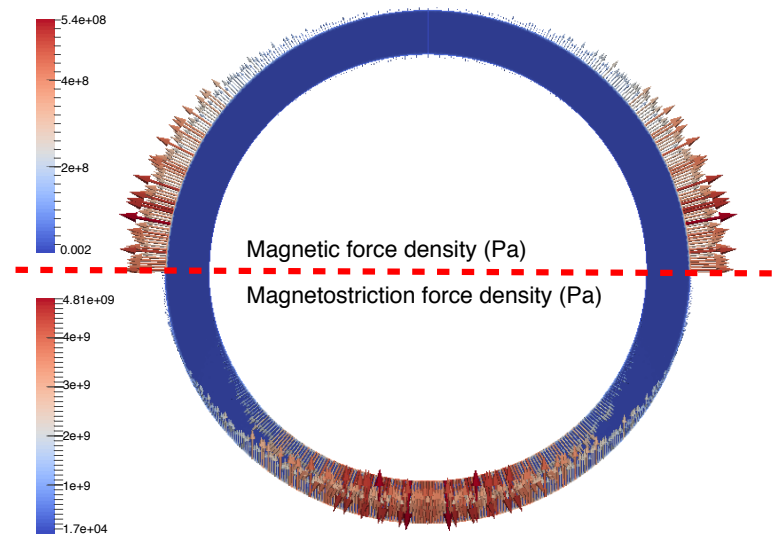

Fig. 3. Magnetic force density (top); Magnetostriction induced force density (bottom)

(VPP) [14]. This forms nodal force directly from magnetic energy. Projections of force density in magneto-elastic coupled problems are also carried out as comparison. Nodal forces are computed in the source mesh for both magnetostriction and magnetic force. As nodal forces are concentrated only at the nodes, this is first transformed into a field of force density before projection. Force density is obtained by dividing the nodal force by its associated surface of each node, leading to a linear system (13).

$$
[S][f]=[F] \quad \text { with } \quad S_{i, j}=\int_{\mathcal{D}_{m e c}} \omega_{i}^{s} \omega_{j}^{s} \mathrm{~d} \tau
$$

where $[F]$ and $[f]$ are respectively the array of nodal force and the degrees of freedom of the force density. Projection of force density is then carried out using formulation (1). Given $\overrightarrow{f^{s}} \in V_{1}$, find $\vec{f}^{t} \in V_{2}$, such that:

$$
\int_{\mathcal{D}_{m e c}} \overrightarrow{f^{s}} \cdot \overrightarrow{f^{\prime}} \mathrm{d} \tau=\int_{\mathcal{D}_{m e c}} \overrightarrow{f^{t}} \cdot \overrightarrow{f^{\prime}} \mathrm{d} \tau, \quad \forall \vec{f}^{\prime} \in V_{2} .
$$

\section{AppliCATION AND RESUlts}

\section{A. Example 1: isotropic case}

The proposed projection formulations are applied in an academic example, in order to compare source tensor projection and force density projection. A ferromagnetic ring made of $\mathrm{FeSi}$ is plunged in the magnetic field. Magnetic potential $\phi$ is set to different values on left and right borders, forming an horizontal magnetic field in computation domain $(\vec{H}=-\operatorname{grad}(\phi))$. Fig.3 gives the distribution of the magnetic force density and magnetostriction equivalent force density computed on a reference well refined mesh. Source tensors and force densities are then computed in source mesh and projected to the target mesh, leading to static mechanical resolution. The fields of displacement due to magnetostriction and magnetic force are finally obtained.

Two meshes are considered: a well refined mesh M1 (144 K elements) and a relatively coarser mesh M2 (36 K elements). M1 and M2 are used respectively as source mesh and target mesh. In order to eliminate the influence of mesh sensitivity, tests are carried out using the same mesh (M1 or M2) without data projection. Similar magnetic and mechanical field are observed on the two meshes.
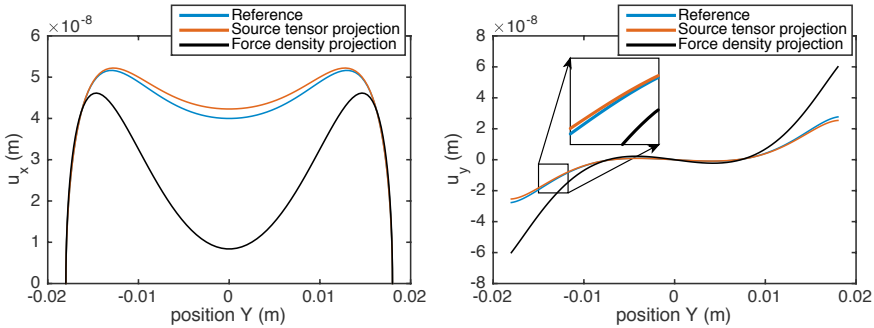

Fig. 4. Displacements due to magnetostriction in direction $\mathrm{X}$ (left) and $\mathrm{Y}$ (right).
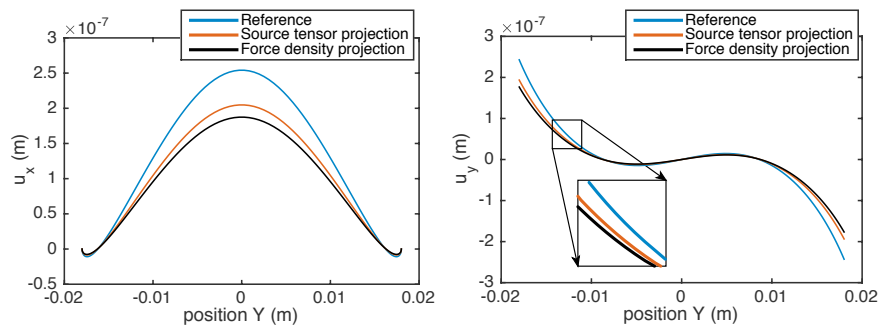

Fig. 5. Displacements due to magnetic force in direction $\mathrm{X}$ (left) and $\mathrm{Y}$ (right).

Displacements of the outer border on the right side of the FeSi ring are shown in Fig.4-5. Fig.4 and Fig.5 are dedicated respectively to magnetostriction and magnetic force induced displacement. Comparisons are made between three different approaches: the same well refined mesh M1 without projections as reference, a coarser mesh M2 for the mechanical resolution with the source tensor projection, as well as the classical force density projection. For magnetostriction induced displacement, source tensor projection shows great efficiency over force density projection. For displacements due to magnetic forces, source tensor projection gives slightly better projection results.

The efficiency of the source tensor projection is different for forces of different physical natures. This can possibly be explained as follows: Free magnetostriction strain exists only in the ferromagnetic material, and is null in the air. However, Maxwell tensor exists both in the air and ferromagnetic material. Magnetic force mainly depends on the discontinuity of the Maxwell tensor on the air-iron interface. This makes magnetic force extremely sensitive to the change of mesh (data projection), which modifies the discontinuity on the interface.

\section{B. Example 2: anisotropic case}

In order to verify the efficiency of the source tensor projection, another example is proposed: multi-layer 'E-I' power transformer core. This core is made of grain-oriented FeSi with strong magnetic and magnetostrictive anisotropy. By modifying the parameters of domain energy, SMSM is able to represent the nonlinear and anisotropic magnetic magnetostrictive behavior. E-shaped and I-shaped sheets are cut along the rolling direction of the lamination, and assembled alternatively from layer to layer, as shown in Fig. 6. Rolling direction of the lamination is indicated by white arrows. The homogenization technique developed in [12] is applied, leading to $2 \mathrm{D}$ modeling of the $3 \mathrm{D}$ structure. In this case, magnetic force is negligible because there is no air-gap. The 

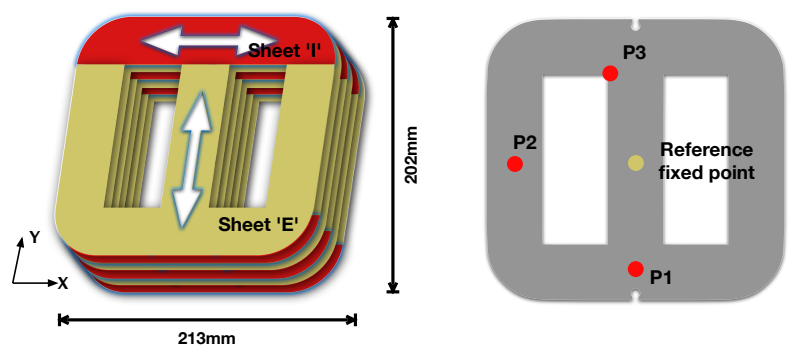

Fig. 6. Multi-layer transformer core structure (left) and measure point (right).
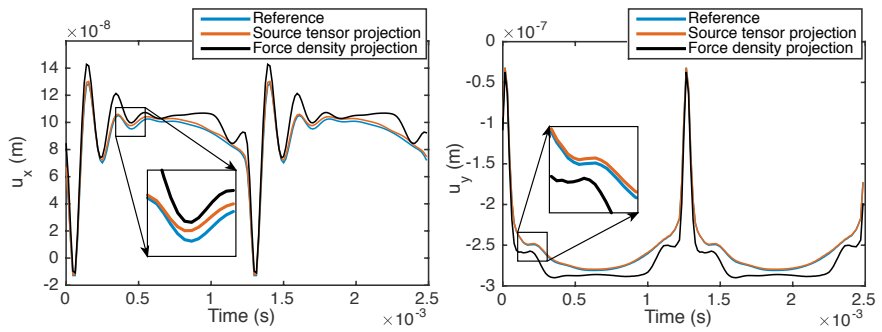

Fig. 7. Displacements in direction $X$ as a function of time at P2 (left); Displacements in direction $\mathrm{Y}$ as a function of time at P1 (right)
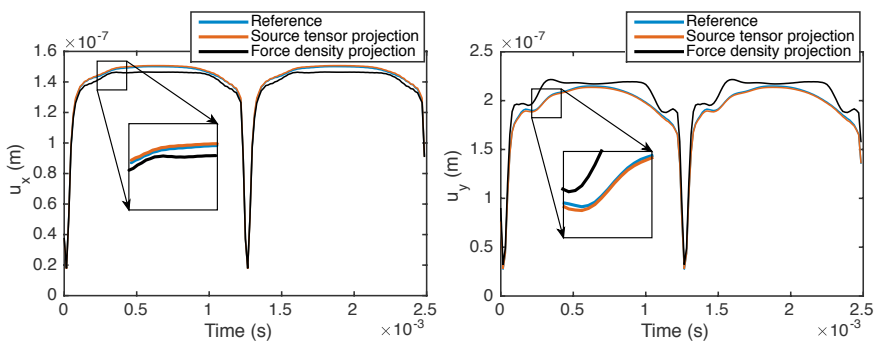

Fig. 8. Displacements in direction $\mathrm{X}$ (left) and $\mathrm{Y}$ (right) as a function of time at P3

efficiency of source tensor projection is only verified for magnetostriction.

Sinusoidal current excitation is set in central limb of the power transformer. By using time step approach, a set of static magnetic problems is solved at different excitation currents. Free magnetostriction strain tensor or magnetostriction induced force are then projected to the target mesh for dynamic mechanical resolution. Dynamic mechanical resolution is carried out in frequency domain, leading to displacement fields of all the harmonics. Displacements at three representative locations (Fig. 6) are shown as functions of time (Fig.7-8).

Excitation current is set in the center coil to $400 \mathrm{~Hz}$ and 200At. By imposing the boundary condition, a fixed point is set as reference in the center of the transformer, as shown in Fig. 6. P1, P2 and P3 are selected as point of measurement. The same comparison procedure as in previous example is applied with M1 (56 K elements) and M2 (14 K elements). Displacements in directions $\mathrm{X}$ and $\mathrm{Y}$ of the point $\mathrm{P} 1, \mathrm{P} 2$ and P3 are shown in Fig.7-8. This numerical example shows better performance of source tensor projection over force density projection in case of magnetostriction induced displacement.

\section{CONCLUSION}

Studies of source tensor projection and force density projection are carried out, based on magneto-elastic modeling chain. By involving SMSM, materials with different magnetic and magnetostrictive behaviors are considered. For magnetostriction, great efficiency of source tensor projection over force density projection is proved in both static and dynamic magneto-elastic problems. For magnetic force, overall better results are observed using source tensor projection. Different efficiencies of source tensor projection for forces of different physical natures are explained.

\section{REFERENCES}

[1] X. Mininger, N. Galopin, X. Ojeda, F. Bouillault, and M. Gabsi, "Modeling of magnetoelastic and piezoelectric coupling: Application to srm noise damping," IEEE Transactions on Magnetics, vol. 45, no. 3, pp. 1218-1221, 2009.

[2] L. Bernard, X. Mininger, L. Daniel, G. Krebs, F. Bouillault, and M. Gabsi, "Effect of stress on switched reluctance motors: a magneto-elastic finite-element approach based on multiscale constitutive laws," IEEE Transactions on Magnetics, vol. 47, no. 9, pp. 2171-2178, sep 2011.

[3] C. Geuzaine, B. Meys, F. Henrotte, P. Dular, and W. Legros, "A Galerkin projection method for mixed finite elements," IEEE Transactions on Magnetics, vol. 35, no. 3, 1999.

[4] P. G. Ciarlet, "Preface," Studies in Mathematics and its Applications, vol. 20, pp. ix-Xv, 1988.

[5] C. W. Rowley, T. Colonius, and R. M. Murray, "Model reduction for compressible flows using POD and Galerkin projection," Physica D: Nonlinear Phenomena, vol. 189, no. 1-2, pp. 115-129, 2004.

[6] P. Nachtergaele, D. J. Rixen, and A. M. Steenhoek, "Efficient weakly coupled projection basis for the reduction of thermo-mechanical models," Journal of Computational and Applied Mathematics, vol. 234, no. 7, pp. 2272-2278, 2010.

[7] G. Parent, P. Dular, J. P. Ducreux, and F. Piriou, "Using a galerkin projection method for coupled problems," IEEE Transactions on Magnetics, vol. 44, no. 6, pp. 830-833, 2008.

[8] Z. Wang, T. Henneron, N. Nemitz, J. C. Mipo, and F. Piriou, "Electromagnetic field projection on finite element overlapping domains," IEEE Transactions on Magnetics, vol. 49, no. 4, pp. 1290-1298, 2013.

[9] Z. Wang, Z. Tang, T. Henneron, F. Piriou, and J.-C. Mipo, "Energetic Galerkin Projection of Electromagnetic Fields Between Different Meshes," IEEE Transactions on Magnetics, vol. 50, no. 2, pp. 613-616, 2014.

[10] A. A. Journeaux, F. Bouillault, and J.-Y. Roger, "Multi-physics problems computation using numerically adapted meshes: application to magneto-thermo-mechanical systems," The European Physical Journal Applied Physics, vol. 61, no. 3, p. 30001, 2013.

[11] L. Bernard and L. Daniel, "Effect of stress on magnetic hysteresis losses in a switched reluctance motor: application to stator and rotor shrink-fitting," IEEE Transactions on Magnetics, vol. 51, no. 9, pp. 1-1, 2015.

[12] M. Liu, O. Hubert, X. Mininger, F. Bouillault, and L. Bernard, "Homogenized magneto-elastic behavior model for the computation of strain due to magnetostriction in transformers," IEEE Transactions on Magnetics, vol. 52, no. 2, pp. 1-12, 2016.

[13] F. Henrotte, "Handbook for the computation of electromagnetic forces in a continuous medium," Int. Compumag Society Newsletter, vol. 24, no. 2, pp. 3-9, 2004.

[14] Z. Ren and A. Razek, "Local force computation in deformable bodies using edge elements," IEEE Transactions on Magnetics, vol. 28, no. 2, pp. 1212-1215, mar 1992. 